\title{
Low Temperature Ageing Behaviour of Quenched Cu-Al-Be Shape Memory Alloy
}

\author{
A. Planes, Ll. Mañosa, M.A. Jurado, R. Romero* and A. Somoza \\ Departament d'Estructura i Constituents de la Matèria, Facultat de Física, Universitat de Barcelona, \\ Diagonal 647, 08028 Barcelona, Catalonia, Spain \\ * IFIMAT, Universidad Nacional del Centro de la Provincia de Buenos Aires, Pinto 399, 7000 Tandil, \\ Argentina, and Comisión de Investigaciones Científicas de la Provincia de Bueno Aires, Argentina
}

\begin{abstract}
Positron lifetime measurements are used to obtain the concentration of quenched-in point defects for different quenching temperatures and to determine their subsequent time evolution at room temperature. The corresponding evolution of the transition temperature after the quench tias also been measured by calorimetry. The fact that both quantities follow comparable time evolutions corroborates the claim that vacancies are responsible for the observed changes in the martensitic transition properties after quenching.
\end{abstract}

\section{INTRODUCTION}

In the last years, there has been an increasing interest in the investigation of how thermodynamic and kinetic properties of martensitic transitions are influenced by quenches from moderate and high temperatures. Most of these studies have been conducted in the $\mathrm{Cu}-\mathrm{Zn}-\mathrm{Al}$ shape memory alloy $[1,2]$. In this system it is acknowledged that the main effect induced by a quench is to retain, at low temperature, a certain degree of configurational disorder and an excess of vacancies. Results indicate that quenched-in disorder modifies the equilibrium between the high ( $\beta$-phase) and low (martensite) phases, resulting in a decrease of the transition temperature $M_{s}$ and of the latent heat; the amount of these decreases depends on quenching temperature $T_{q}$. Quenched-in vacancies are of fundamental importance in relation with the post-quench behaviour of $\mathrm{Cu}$-based alloys. Indeed, after a quench there is an evolution towards equilibrium, essentially associated with an ordering process that involves the exchange of atoms among the different lattice sites via a vacancy mechanism [3]. Since vacancies are eliminated during ageing and also their mobility is affected by the development of atomic order, the process appears to be a complex one [4].

In this work we are interested in the $\mathrm{Cu}-\mathrm{Al}-\mathrm{Be}$ shape memory alloy. This alloy belongs to the same family of $\mathrm{Cu}-\mathrm{Zn}$ - $\mathrm{Al}$ (Hume-Rothery alloys) but in contrast with $\mathrm{Cu}-\mathrm{Zn}-\mathrm{Al}$, it undergoes a single firstorder order-disorder transition at a relatively high temperature $(\sim 800 \mathrm{~K})$ [5]. This is the reason why it is practically impossible to modify the low temperature long range ordered structure by means of a quench. Even so, the properties of the martensitic transition are known to be affected by quenching. Actually an increase in the transition temperature and in the latent heat after the quench has been reported. This has been recently accounted for by assuming that these modifications are associated to an effective change of Be-concentration, induced by an excess of vacancies [6]. In this paper we present positron annihilation and calorimetric measurements of the time evolution of vacancy concentration and martensitic transition temperature, during the post-quench ageing process. These experiments are aimed to gain a deeper insight into the problem of quenches in $\mathrm{Cu}-\mathrm{Al}-\mathrm{Be}$. 


\section{EXPERIMENTAL}

Measurements have been performed on a $\mathrm{Cu}-\mathrm{Al}-\mathrm{Be}$ single crystal with composition $\mathrm{Cu} ; 22.72$ at $\%$ $\mathrm{Al} ; 3.55$ at\% Be. At high temperature this system undergoes an order-disorder transition from a disordered to a $\mathrm{DO}_{3}$ structure at $\mathrm{T}_{c}=793 \mathrm{~K}$. The nominal Ms temperature is $145 \mathrm{~K}$.

Samples of $15 \mathrm{~mm}$ diameter and 1.5 thickness for positron and calorimetric measurements have been cut from the original rod using a low speed diamond saw. The damaged surface was etched away with a solution of $50 \% \mathrm{HNO}_{3}$ in water. All the specimens were first maintained at $1073 \mathrm{~K}$ for $1800 \mathrm{~s}$ and subsequently air cooled down to a temperature $\mathrm{T}_{q}$ from which they are quenched in an ice-water mixture. The quenching temperatures covered the range from $473 \mathrm{~K}$ up to $1073 \mathrm{~K}$. In all cases (positron and calorimetric measurements), the total elapsed time between the quench and the beginning of measurements was typically $\sim 15 \mathrm{~min}$.

For positron measurements, a $20 \mu \mathrm{Ci}$ source of ${ }^{22} \mathrm{NaCl}$ deposited on a thin kapton foil (1.1 $\mathrm{mg} / \mathrm{cm}^{2}$ ) was sandwiched between two identical specimens. The lifetime spectrometer was a fast-fast timing coincidence system with a resolution of $255 \mathrm{ps.} \mathrm{Calorimetric} \mathrm{measurements} \mathrm{were} \mathrm{conducted}$ using a microcalorimeter equipped with semi-conducting thermoelectric power elements as heat-flow transducers mounted differentially on top of a copper block. A four point Pt-100 resistance embedded in the copper block was used to monitor the temperature.

\section{RESULTS}

Positron results have been characterized by means of two lifetime components $\tau_{1}$ and $\tau_{2}$. After quenching, for all studied $\mathrm{T}_{q}$, we have obtained that the long-living component $\tau_{2}$ takes always, within the errors, the same value and that $\langle\tau\rangle\left(=I_{1} \tau_{1}+I_{2} \tau_{2}\right.$, being $\left.I_{1}+I_{2}=1\right)$ and $I_{2}$ both decrease during the ageing process. Typical results for $\mathrm{T}_{q}=1073 \mathrm{~K}$ are displayed in fig.1. According to the well-known two-state standard trapping model (STM) [7] a decrease with time of the relative intensity $I_{2}$ is related to a decrease in the defect concentration in the system and a decrease of the mean lifetime $\langle\tau\rangle$, to the migration of defects. Consistently with the STM, we have observed that in all cases $\tau_{1}$ increases with ageing. From these results, it is reasonable to assume that quenched-in defects are vacancies. Hence, in what follows a suffix "v" will be used to identify the second lifetime.

Within the two-state standard trapping model the concentration $\mathrm{C}_{v}$ of vacancies is given by [8]:

$$
K_{v=\mu_{v}} C_{v}=\frac{1}{\tau_{f}}\left\{\frac{\langle\tau\rangle-\tau_{f}}{\tau_{v}-\langle\tau\rangle}\right\}
$$

where $\tau_{f}$ is the positron lifetime in the bulk state and $\mu_{v}$ is the specific trapping rate for positrons in a material with bcc structure. A value of $\tau_{f}=119 \pm 1$ ps has been evaluated following the method described in [9] with $\mu_{v}=10^{15} \mathrm{~s}^{-1}$ as proposed in [10]. The concentration of vacancies just after the quench $\mathrm{C}_{v}(0)$, has been evaluated from a linear extrapolation at $\mathrm{t}=0$ of the first non-saturated values of $\ln \left(\tau_{f} \mathrm{~K}_{v}\right)$. The obtained dependence of the concentration of vacancies immediatly after quench as a function of $\mathrm{T}_{q}$ is plotted in fig.2. The large statistical scatter around points corresponding to higher vacancy concentrations originates from the long time that elapses after quenching, during which the positron signal remains in the saturation regime. In spite of that, it is clear that in the range of quenching temperatures from 650 to $770 \mathrm{~K}$, the initial concentrations of quenched-in vacancies are about 5 to 10 times the concentration retained for other $\mathrm{T}_{q}$ temperatures. The post-quench time evolution of the vacancy excess is displayed in figures $3 \mathrm{a}$ and $3 \mathrm{~b}$ for $\mathrm{T}_{q}=773 \mathrm{~K}$ and $\mathrm{T}_{q}=1073 \mathrm{~K}$ respectively (open symbols). 


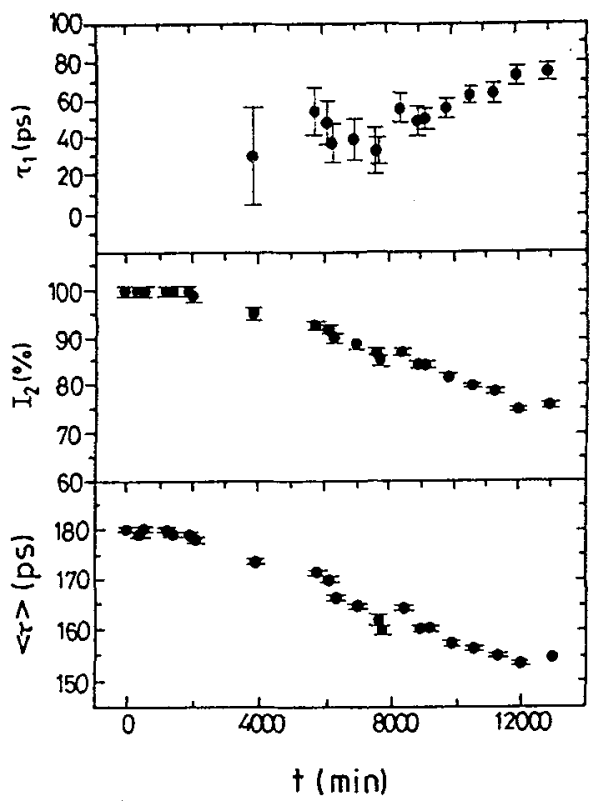

Figure 1 Positron lifetime parameters $\tau_{1}, \mathrm{I}_{2}$ and $\langle\tau\rangle$ as a function of ageing time at room temperature for $\mathrm{T}_{q}=1073 \mathrm{~K}$.

Calorimetric measurements show that forward and reverse martensitic transition temperatures are also considerably affected by a quench. We have quantified the amount of change in these temperatures as a function of $\mathrm{Tq}$, by means of the temperatures $\mathrm{T}_{M}$ and $\mathrm{T}_{A}$ corresponding to the maxima of the calorimetric peaks recorded in the forward and reverse transitions respectively. These temperatures, measured immediately after the quench, are plotted in fig. 4 as a function of $\mathrm{T}_{q}$. The values found for these temperatures first increase until around $650 \mathrm{~K}$, then decrease until around $850 \mathrm{~K}$ where the curve exhibits a minimum, and increase again for larger $\mathrm{T}_{q}$. It is worth mentioning that, within the experimental errors, the transition entropy change is independent of $T_{q}$. This proves that changes in transition temperatures and latent heats are proportional. The post-quench time evolution of $\mathrm{T}_{A}$ has been measured for $\mathrm{T}_{q}=773 \mathrm{~K}$ and $\mathrm{T}_{q}=1073 \mathrm{~K}$. Results are shown in figs. 3a and $3 \mathrm{~b}$, respectively (solid symbols).

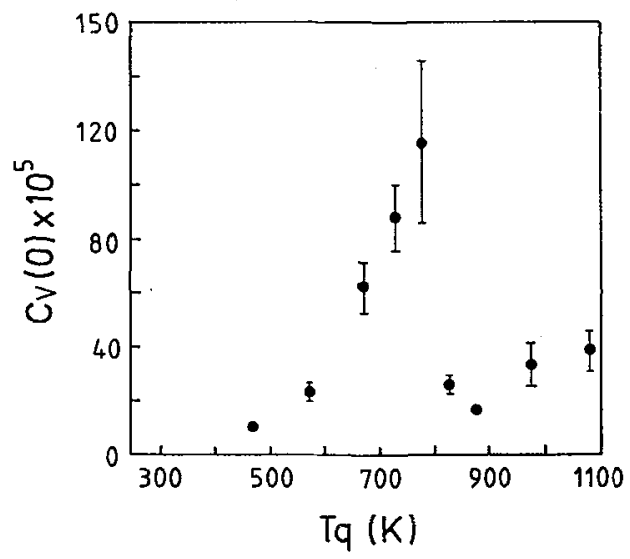

Figure 2 Initial concentration of quenched-in vacancies $\mathrm{C}_{v}(0)$ as a function of $\mathrm{T}_{q}$. 

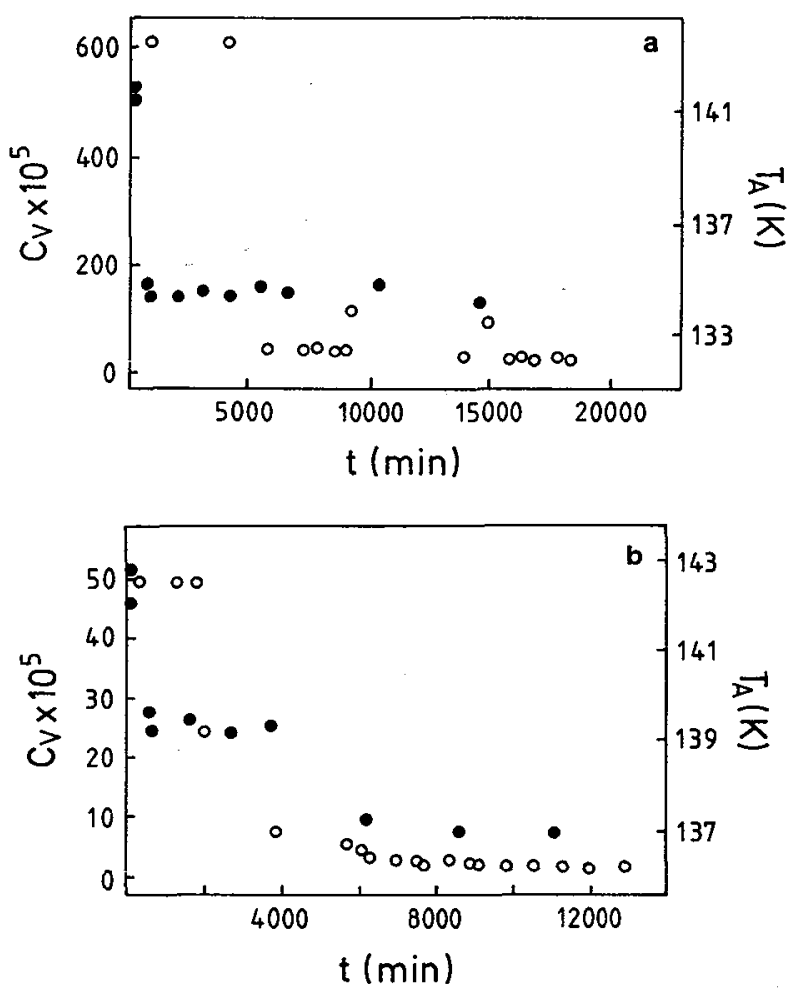

Figure 3 Post-quench time evolution of the excess of vacancy (open symbols) and of the reverse martensitic transition temperature $\mathrm{T}_{A}$ (solid symbols). (a) $\mathrm{T}_{q}=773 \mathrm{~K}$, (b) $\mathrm{T}_{q}=1073 \mathrm{~K}$.

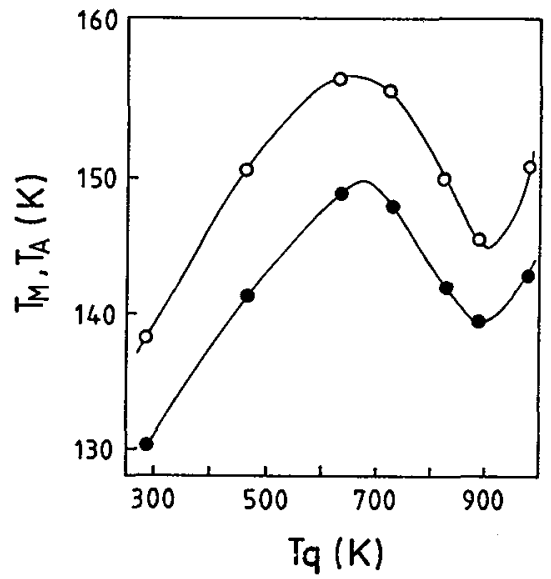

Figure 4 Dependence with $\mathrm{T}_{q}$ of the temperatures $\mathrm{T}_{A}$ (open symbols) and $\mathrm{T}_{M}$ (solid symbols) measured immediately after quenching. These temperatures are given within $\pm 2 \mathrm{~K}$. Lines are guides to the eye. 


\section{DISCUSSION}

In this work we have obtained the excess of vacancies and the change in the martensitic transition temperature in $\mathrm{Cu}-\mathrm{Al}-\mathrm{Be}$ as a function of quenching temperature $\mathrm{T}_{q}$, by means of positron lifetime and calorimetric measurements. Since it is known that in this system the degree of atomic order is not altered by quenching [11], any modification in the relative phase stability between $\beta$ and martensitic phases is likely to be due to an excess of quenched-in vacancies. The effect of vacancies on the martensitic transition temperature can be understood by assuming that vacancies induce a change in the actual concentration of $\mathrm{Be}$. Actually, it is known that $\mathrm{Be}$ atoms are distributed randomly (at least for low Be concentrations) among the different lattice sites in the ordered $\mathrm{Cu}$-Al-Be system [5]. Since vacancies also distribute randomly, we assume that they substitute Be atoms. Hence, the change in the Be concentration after quenching from a given $\mathrm{T}_{q}$ is expected to be:

$$
\delta C_{B e} \sim-C_{v}(0)
$$

where we have considered that $\mathrm{C}_{v}(0) \gg \mathrm{C}_{v}^{e q}$, with $\mathrm{C}_{v}^{e q}$, the equilibrium vacancy concentration at room temperature. In the above expression $\mathrm{C}_{B e}$ is the $\mathrm{Be}$ concentration and $\mathrm{C}_{v}(0)$, the quenched-in vacancy concentration just after the quench from $\mathrm{T}_{q}$. Now it is important to remember that in $\mathrm{Cu}-\mathrm{Al}-\mathrm{Be}$, an increase of $1 \%$ in the concentration of Be results in a depletion in the martensitic transition temperature of around $130 \mathrm{~K}[12]$. For $\mathrm{T}_{q} \sim 800 \mathrm{~K}, \mathrm{C}_{v}(0) \sim 10^{-3}$ (maximum concentration of quenched-in vacancies) which should lead to an increase in the transition temperature $>10 \mathrm{~K}$. This prediction is in very good agreement with the calorimetrically measured shifts in the transition temperature (see fig.4). In addition, it is worth noting that transition temperature and quenched-in vacancy concentration follow, as a function of $T_{q}$, similar behaviour.

The suggestion that the excess of quenched-in vacancies are responsible for the shifts in the transition temperatures after a quench, is corroborated by the results obtained in the post-quench ageing process. As shown in figures 3 , the qualitative behaviour of these two quantities is similar. After a quench from $T_{q}=773 \mathrm{~K}$ (fig. 3a), transition temperatures decreases sharply in the first $700 \mathrm{~min}$, and remain almost unaltered during the ulterior ageing; on the other hand, the vacancy concentration does not evolve significantly for ageing time longer than $5000 \mathrm{~min}$, and the decrease in the early stages is undetectable because the concentration of vacancies is above the detection limit, resulting in a saturation of the positron signal [6]. After the quench from $\mathrm{T}_{q}=1073 \mathrm{~K}$ (fig. $3 \mathrm{~b}$ ), the correlation betweeen transition temperature and vacancy concentration is both qualitative and quantitative; in a first stage $(t<2000 \mathrm{~min}), \mathrm{T}_{A}$ (and $\mathrm{T}_{M}$ ) exhibits a significant decrease while the vacancy concentration is in its saturation regime; this stage is followed by an approximately exponential decrease in both quantities. A time constant of $t=2270 \mathrm{~min}$ fits reasonably well the experimental values obtained for the transition temperatures and for the concentration of vacancies.

\section{Acknowledgments}

This work has received financial support from the CICyT (Spain), project MAT95-0504, from the CIRIT (Catalonia), project SGR119 and from Consejo Nacional de Investigaciones Científicas y Técnicas, Comisión de Investigaciones de la Provincia de Buenos Aires and Secretaría de Ciencia y Técnica de la UNCentro (Argentina) 


\section{References}

[1] R. Rapacioli, M. Ahlers, Acta metall., 27, 777 (1979)

[2] A. Planes, J.L. Macqueron, R. Rapacioli, G. Guénin, Phil. Mag. A, 61, 221 (1990)

[3] T. Suzuki, Y. Fugii, A. Nagasawa, Mater. Sci. Forum, 56-58, 481 (1990)

[4] J. Elgueta, J.L. Macqueron, A. Planes, J. Phys. Condens. Matter, 4, 285 (1992)

[5] M.A. Jurado, T. Castán, Ll. Mañosa, A. Planes, J. Bassas, X. Alcobé, M. Morin, Phil. Mag. A, 75, 1237 (1997)

[6] R. Romero, A. Somoza, M.A. Jurado, A. Planes, Ll. Mañosa, Acta mater., 45, 2101 (1997)

[7] M. Franz, Th. Hehenkamp, Th. Kluin, J.D. McGervey, Phys. Rev. B, 48, 3507 (1993)

[8] H.E. Schaefer, phys. stat. solidi (a), 102, 47 (1987)

[9] F. Plazaola, R. Romero, A. Somoza, Nuovo Cimento D, 1997

[10] P. Hautojärvi, Mater Sci. Forum, 15-16, 81 (1993)

[11] Ll. Mañosa, J. Zarestky, C. Stassis, 1996, unpublished results

[12] S. Belkhala, G. Guénin, J. Phys. IV, 1, C4-145 (1991) 